\title{
A Systematic Review and Meta-Analysis on the Efficacy of Curcumin/Turmeric for the Prevention and Amelioration of Radiotherapy/Radiochemotherapy Induced Oral Mucositis in Head and Neck Cancer Patients
}

\author{
Sreedevi Dharman ${ }^{1 *}$, Maragathavalli $\mathbf{G}^{1}$, Karpagavalli Shanmugasundaram ${ }^{2}$, \\ Rajesh Kumar Shanmugam ${ }^{3}$
}

\begin{abstract}
Background: Oral Mucositis(OM) is an acute debilitating dose limiting toxicity of Radiotherapy/ Radiochemotherapy(RT/RCT) in management of Head and Neck Cancer (HNC). Curcumin/Turmeric may reduce OM in patients. Aim: Efficacy of Curcumin/Turmeric for preventing and ameliorating the onset and severity of RT/RCT induced OM was analysed in this review. Methods: A systematic literature search with meta-analysis were performed using Mesh terms in PubMed, Google scholar, Science Direct, Cochrane library and manual searching, articles published from 2010 to April 2021 were included. Clinical trials that studied the efficacy/effects of turmeric / curcumin in management of RT/RCT induced OM in HNC patients were included. Statistical Analysis were done to calculate the pooled Risk ratio at $95 \%$ confidence interval with significance at $p<0.05$. Results: Nine studies with overall 582 patients with HNC undergoing RT/RCT were included qualitatively. From evidence, orally 1,500-2,000 mg/day of Curcumin/Turmeric, $(80 \mathrm{mg} / \mathrm{day} / 0.1 \%$ mouthwash) of nanocurcumin, topically gel $/$ mouthwash used with increase in frequency prior and the entire course of RT/RCT with long follow ups are beneficial with no serious adverse effects. Meta-analysis of 5 prophylactic trials favoured curcumin/turmeric in reducing the severity of OM (RR 0.48 at $95 \%$ $\mathrm{CI}=0.23,0.99, \mathrm{P}=0.05)$, did not prevent the overall incidence of $\mathrm{OM}(\mathrm{RR} 0.99$ at $95 \% \mathrm{CI}=0.95,1.03, \mathrm{P}=0.67)$ but delayed the onset of OM (RR 0.38 at $95 \% \mathrm{CI}=0.18,0.80, \mathrm{P}=0.01$ ) during $\mathrm{RT} / \mathrm{RCT}$ compared to control. Mean mucositis grade (Grade 3) was reduced in curcumin/turmeric with a mean difference of $(-0.85$ at $95 \% \mathrm{CI}=-1.02,0.67, \mathrm{P}<0.00001)$ over control. Pooling of 2 therapeutic trials favoured Curcumin/Turmeric with significant reduction of pain score at a mean difference of -2.17 at $95 \% \mathrm{CI}=-2.77,-1.58, \mathrm{P}<0.00001$ over chlorhexidine. Conclusion: Curcumin/Turmeric are safe, efficacious, well tolerated in preventing the delay in onset and severity of OM, therapeutically ameliorated pain in patients with cancer therapy induced OM. We recommend novel curcumin formulations, high quality randomised controlled trials to further improve its therapeutic effects.
\end{abstract}

Keywords: Turmeric- curcumin- oral mucositis- radiotherapy- radio-chemotherapy- oral cancer- systematic review

Asian Pac J Cancer Prev, 22 (6), 1671-1684

\section{Introduction}

Systemic chemotherapy, radiotherapy or both have become more effective for managing head and neck carcinoma, but they are related with short and long term side-effects (Paiar et al., 2020). Oral mucositis is a common short term side effect that are painful inflammation and ulceration of oral-pharyngeal mucosa (Sonis et al., 2004).

There is functional disruption and integrity of oral mucosa that are acute and noticed as redness to severe ulceration and are infected by fungus such as oral candidiasis (Yuan and Sonis., 2014). High dose chemotherapy induced oral mucositis produce atrophy of the mucosal lining of mouth followed by ulcer formation affecting $100 \%$ of patients, radiotherapy induced oral mucositis frequently affecting upto $80 \%$ of patients, conventional chemotherapy upto $20-40 \%$ (Lalla et al., 2014). Mouth soreness with erythema occurs within 2 weeks of start of radiotherapy, followed by severe epithelial damage within next 2 weeks (Duncan et al., 2005). With concurrent chemoradiotherapy, incidence and severity

${ }^{1}$ Department of Oral Medicine and Radiology, Saveetha Dental College and Hospitals, Saveetha Institute of Medical and Technical Sciences, Saveetha University, Chennai, Tamil Nadu, India. ${ }^{2}$ Department of Oral Medicine and Radiology, Seema Dental College and Hospitals, Rishikesh, India. ${ }^{3}$ Department of Pharmacology, Saveetha Dental College, Saveetha Institute of Medical and Technical Sciences, Saveetha University, Chennai, India.*For Correspondence: sanjamrut@gmail.com 
of OM are still greater (Ver-Llonch et al., 2006). These cancer treatments produced complications causes pain or oral irritation such as difficulty in swallowing and eating, bleeding, reduced nutrition, leading to weight loss, deferral in cancer therapy, extended hospitalisation, expenses and life frightening infections such as septicaemia (Bowen et al., 2019, Murphy et al., 2007).

Based on the severity, OM is classified as tolerable mucositis (grade 1 and 2 mucositis) and intolerable mucositis (grade 3 or more) (Khanal et al., 2010). In $\mathrm{HNC}$ undergoing radiotherapy, tolerable mucositis begins in all patients and is manageable, intolerable mucositis requires effective pain management, gastrostomy tube, IV line for nutritional supplementation (Elting et al., 2008). This can also inturn lead to radiotherapy reduction and delay in dose, even ending the planned radiotherapy, thus complicating the underlying cancer therapy (Bensinger et al., 2008).

Presently available treatment are only palliative and for intolerable mucositis not widely accepted treatment is available (Rodriguez-Caballero et al., 2012). Chlorhexidine gluconate is frequently used mouthwash solutions, but literatures doesn't support much of its use due to its stinging and dehydration, leading to microbial colonisation further increasing the patient's complication (Cardona et al., 2017). Pain due to OM leads to swallowing difficulty and requires opioid analgesia, which rapidly develop tolerance (Epstein et al., 2019). Honey applied topically inspite of its effectiveness could enhance radiation-related caries (Van den Wyngaert, 2012). Interventions such as basic oral care (Hong et al., 2019), cytokines and growth factors (Logan et al., 2020), cryotherapy (Correa et al., 2020) are suggested by Multinational Association of Supportive Care in Cancer/The International Society of Oral Oncology (MASCC/ISOO) Clinical Practice Guidelines, but none of them were proved to be used as standard treatment. Palifermin (keratinocyte growth factor-1) has been approved by the US Food and Drug Administration but due to its application only for patients on high dose chemotherapy, expensiveness, difficulty to administer, is not considered a better choice (Nooka et al., 2014). Oral zinc sulfate (Tian et al., 2018), Oral glutamine (Anderson and Lalla, 2020), laser therapy (de lima et al., 2020), photobiomodulation therapy (Campos et al., 2020) are recently been tried but definitive therapy has not been established.

Herbal therapy with varied pharmacological benefits with minimal adverse effects are required. Turmeric (Curcuma longa) belongs to Zingiberaceae is a medicinal herb, its active component being curcumin. Curcumin/ Turmeric has antioxidant, analgesic, anti-inflammatory, antimicrobial, wound healing agent, has chemosensitizing and radiosensitizing properties (Nagpal and Sood, 2013). Previous studies have already shown that it is effective against proinflammatory cytokines, cyclooxygenase, prostaglandin E. (Maziero et al., 2018) Turmeric stands forefront in wound healing (Mohanty et al., 2017) Studies have proven turmeric/curcumin are effective in potentially malignant disorders like oral submucous fibrosis (Rai et al., 2019), oral lichen planus (Nosratzehi., 2018)
A systematic review (Normando et al., 2019) due to few studies and heterogeneity was present among studies meta-analysis wasn't performed. A recent meta-analysis reported incidence of severity of oral mucositis(>grade2) alone and subgroup analysis weren't performed (Zhang et al., 2020). The present systematic review highlights the suggested dosage and appropriate usage, bioavailability aspects of turmeric /curcumin in chemoradiotherapy induced oral mucositis in Head and neck cancer patients. Moreover, three studies have been included two for qualitative synthesis and one published recently for quantitative analysis in our review. Prophylactic studies were analysed into Radiotherapy and Radiochemotherapy, topical and oral mainly to compare the curcumin effects across different subgroups, delay in onset (Grade 1) was also performed to assess the prophylactic effect of curcumin. It was aimed to analyse the existing literature on the efficacy of Curcumin/Turmeric for preventing and mitigating oral mucositis in patients undergoing Chemo-radio therapy for head and neck cancers.

\section{Materials and Methods}

\section{Methods}

This systematic review followed PRISMA (Preferred Reporting Items for Systematic Review and Meta-analysis) statement.

\section{Data Source and Search Criteria}

A systematic literature search was done to identify articles describing turmeric/curcumin in $\mathrm{OM}$ on chemo/radiotherapy in HNC patients from 2010 till April 2021 were included. Databases searched were PubMed using MeSH terms, science direct, Cochrane library, google scholar. The search methodology applied in PubMed using MeSH terms with following keywords are, $(()(()(()(()(()((“) C u r c u m i n ”[M e S H$ Terms $]$ OR "Curcumin" [All Fields]) OR "curcumin s" [All fields]) OR"curcumine"[AllFields])OR"curcumins"[AllFields]) OR((("Curcuma"[MeshTerms]OR"Curcuma"[AllFields] OR “curcumas"[AllFields]OR"curcumae"[AllFields ]) )“Curcuma" [MeSH Terms] OR(( "Curcuma"[All Fields]) "Curcuma"[MeSH Terms] OR "Curcuma" [All Fields] OR "turmeric"[All Fields] OR "turmeric extract"[Supplementary Concept]) AND (( ( "Radiotherapy"[MeSH Terms OR "Radiotherapy"[All Fields] OR "radiotherapies" "[All Fields]OR"Radiotherapy"[MeshSubheading] O R "radiotherapys" [ A $11 \mathrm{Fields}])$ ) OR"Radiotherapy"[MeSHTerms])OR((()((“chemoth erapys"[AllFields]OR"drugtherapy"[MeSHTerms]) OR "drug" [All Fields] AND "therapy"[All Fields])) OR "drug therapy" "[MeSH Subheading]) OR "chemotherapy"[AllFields]))OR"chemotherapy,adjuva nt”[MeSHTerms])OR((“Chemotherapy"MeSHTerms] O R "Chemoradiotherapy"[AllFields] OR"chemoradiotherapies"[All Fields])) OR "Chemoradiotherapy"[MeSH Terms]AND(((("Head and Neck Neoplasms"[MeSH Terms]OR((“head”[All Fields] AND "neck" "[All Fields] AND "neoplasms" "[All Fields]))OR "Head and Neck Neoplasms"[All Fields]) 
OR (("head" "[All Fields] AND "neck" "[All Fields] AND "cancer" "[All Fields] or "head and neck cancer" OR "Head and Neck Neoplasms"[MeSH Terms])AND (("stomatitis"[MeSH Terms\} OR "stomatitis[All Fields] OR ("oral” [All Fields]AND "mucositis" [All Fields])) OR “oral mucositis:[All Fields].

The review search included published and unpublished articles and only those listed in English literature. Manual searches of the articles were also performed. The search results were short listed using preset inclusion and exclusion criteria. The articles were screened on the basis of title and abstract. Full text was then procured for the relevant articles which fulfilled the inclusion criteria. Two reviewers searched and analysed the studies independently. Disagreement between articles was discussed and resolved.

\section{Eligibility Criteria}

Inclusion Criteria:

Type of Participants: Prevention/Treatment of oral mucositis undergoing radio-chemotherapy in HNC patients.

Types of Interventions: Curcumin/Turmeric

Comparators: Placebo/Other standardised interventions

Type of Studies: Studies with randomised clinical trials, non randomised clinical trials.

Outcome Measures:

Primary Outcome: Incidence of OM and its severity, Delay in onset of OM, Mean Mucositis grade

Secondary Outcome: Mean pain scores, loss of weight

Pain assessment by Visual Analog Scale(VAS), Numeric Rating Scale(NRS), Oral mucositis is assessed by graded scales such as Radiotherapy Oncology Group (RTOG), World Health Association (WHO) scales, Oral Mucositis Assessment Scale (OMAS), National Cancer Institute Common Toxicity Criteria version 2 scale (NCI-CTC v.2), National Cancer Institute Common Terminology Criteria for Adverse Events, version 4.0(NCI CTCAE assessment V.4)

Onset of mucositis-Time of appearance of first sign of mucositis

In RTOG and WHO scales, grade 1 and 2 are tolerable mucositis, degree of severity of mucositis are grade 3 and above which are intolerable type.

Dropouts-Within number of days the patient had to stop the cancer treatment due to mucositis

Weight loss-Weight loss calculated after the completion of therapy.

\section{Exclusion Criteria}

Animal study, invitro, Editorials/commentaries, surveys, reviews, guidelines were excluded from this systematic review.

\section{Evaluation of methodological quality}

Nine articles were assessed for their quality using Review manager software using Revman 5.4. Quality assessment of interventional studies include seven domains. Each domain consisted of one question which were answered "low risk" or "high risk" or "unclear risk".
The domains included are Random sequence generation (selection bias), Allocation concealment (selection bias), Blinding of participants and personnel (performance bias), Blinding of outcome assessment (detection bias), Incomplete outcome data (attrition bias), Selective reporting (reporting bias) and Other bias. This data was fed into Review manager software namely in Revman 5.4 to obtain a colour coded chart of risk of bias summary and graph.

\section{Data Gathering and synthesis}

Data extraction included author, year, study design, Patients gender, age, sample size, groups, grading scales, curcumin formulations, its dosage and directions, results, adverse effects, outcomes. These data were extracted by 2 authors and discussions were done to rule out any differences.

\section{Statistical Analysis}

Meta-analysis was done using program Review Manager 5.4.1. Pooled effects are calculated when the dichotomous data will be expressed as Relative Risk with $95 \% \mathrm{CI}$, continuous data as mean difference with $95 \% \mathrm{CI}$. $\mathrm{I}^{2}$ method are used to assess the heterogeneity among studies. Heterogeneity was statistically significant if $p$ value was $<0.1$. $\mathrm{I}^{2}$ values of $<25,>25-<75,>75$ suggest low, moderate, high heterogeneity. If heterogeneity was present random effects model was used, if absent fixed effects model are used for overall effects calculation. Funnel Plot was used for the analysis of publication bias

\section{Results}

\section{Selection Criteria}

Initial search strategy on PubMed, Google scholar, Science Direct, Cochrane library and gray literature yielded a total of 2,394 articles based on keywords. Number of articles screened by title and abstract were 1,728 after removing duplicates. Records excluded which did not meet the preset criteria based on their abstracts were 1,711. Full text reviewed for eligibility were 21 , from which 12 articles were removed for not meeting inclusion criteria. 9 articles totally were finalized for this systematic review based on the entire content of the articles. Only seven of the nine studies with 283 participants were included in this review provided data for meta-analysis on overall incidence of onset and severity of oral mucositis.

Figure 1 depicts Prisma Flow chart showing the sources and the final short listed articles included in this review.

\section{Study Design and Duration of included studies}

Seven randomized controlled trials (Rao et al., 2014, Mansourian et al., 2015, Patil et al., 2015, Charanthimath, 2016, Delavarian et al., 2019, Arun et al., 2020, Shah et al., 2021) non randomised controlled trials (Adhvaryu et al., 2018, Saldanha and Almeida, 2014) investigated the efficacy/effects of curcumin in oral mucositis. Evaluation period varied from 5 days to 8 weeks, follow up visits were 5 days (Saldanha and Almeida, 2014), 2 weeks (Charanthimath, 2016), 20 days (Patil et al., 2015), 42 


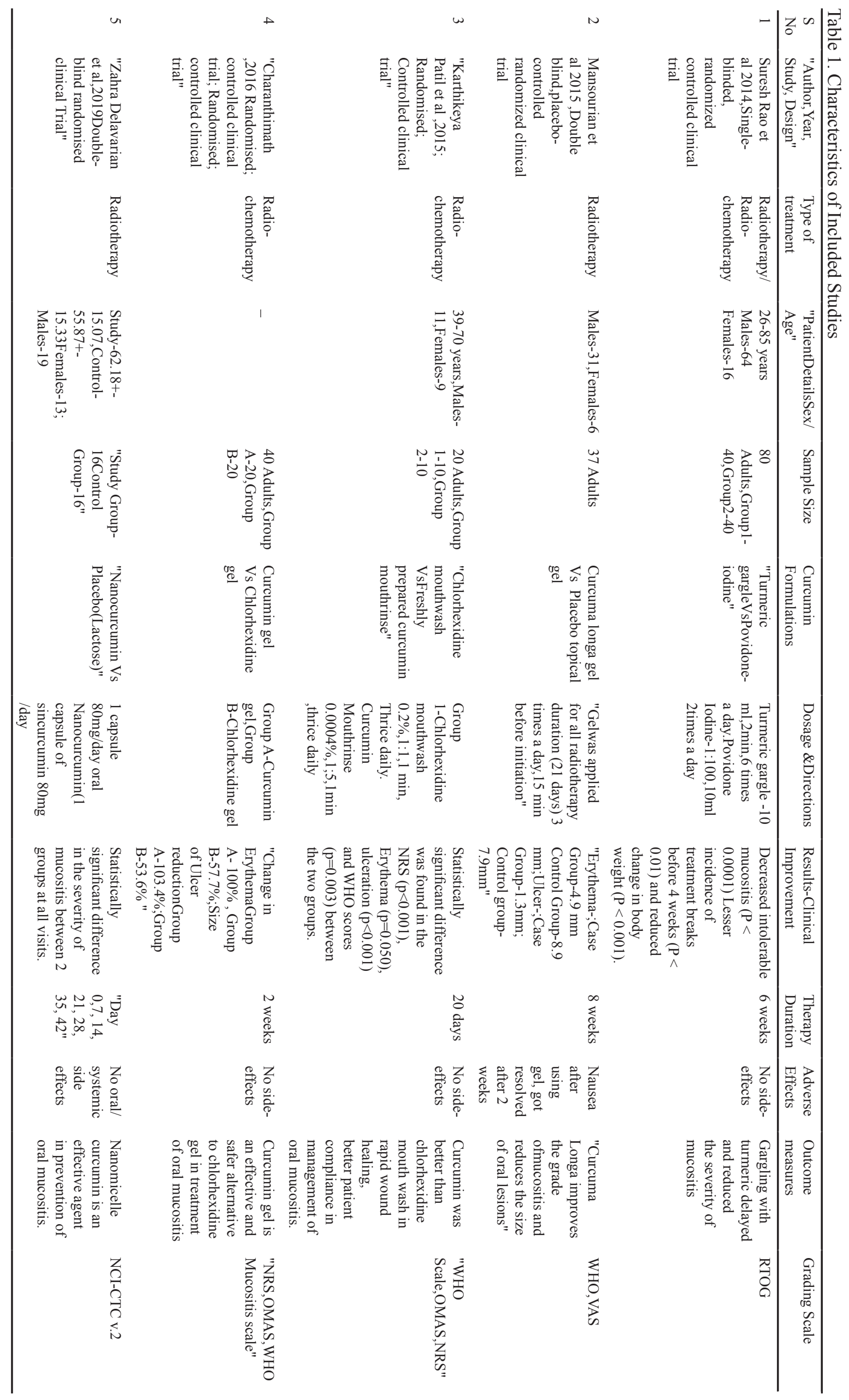


DOI:10.31557/APJCP.2021.22.6.1671

Turmeric/Curcumin Efficacy on Oral Mucositis

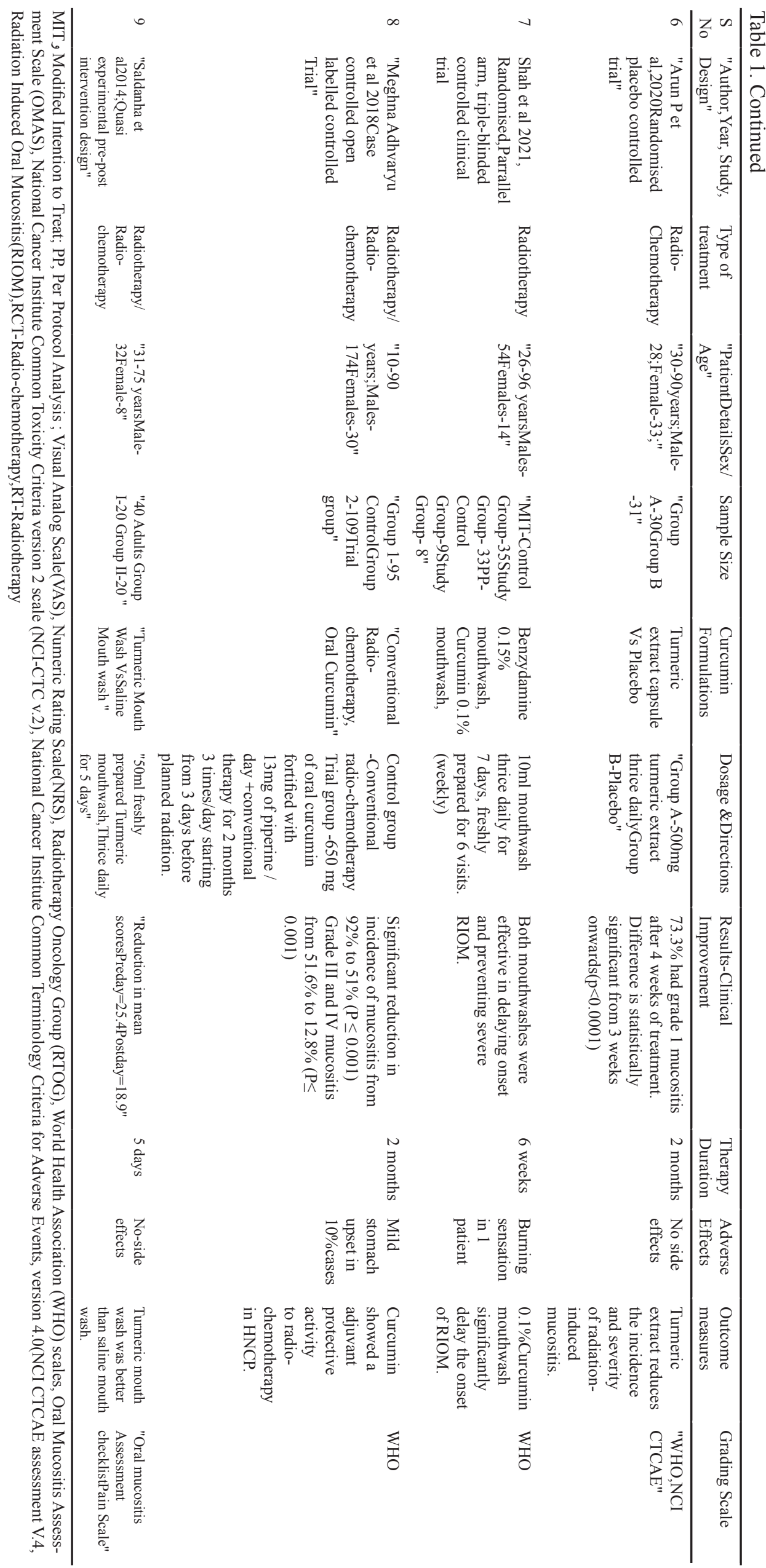




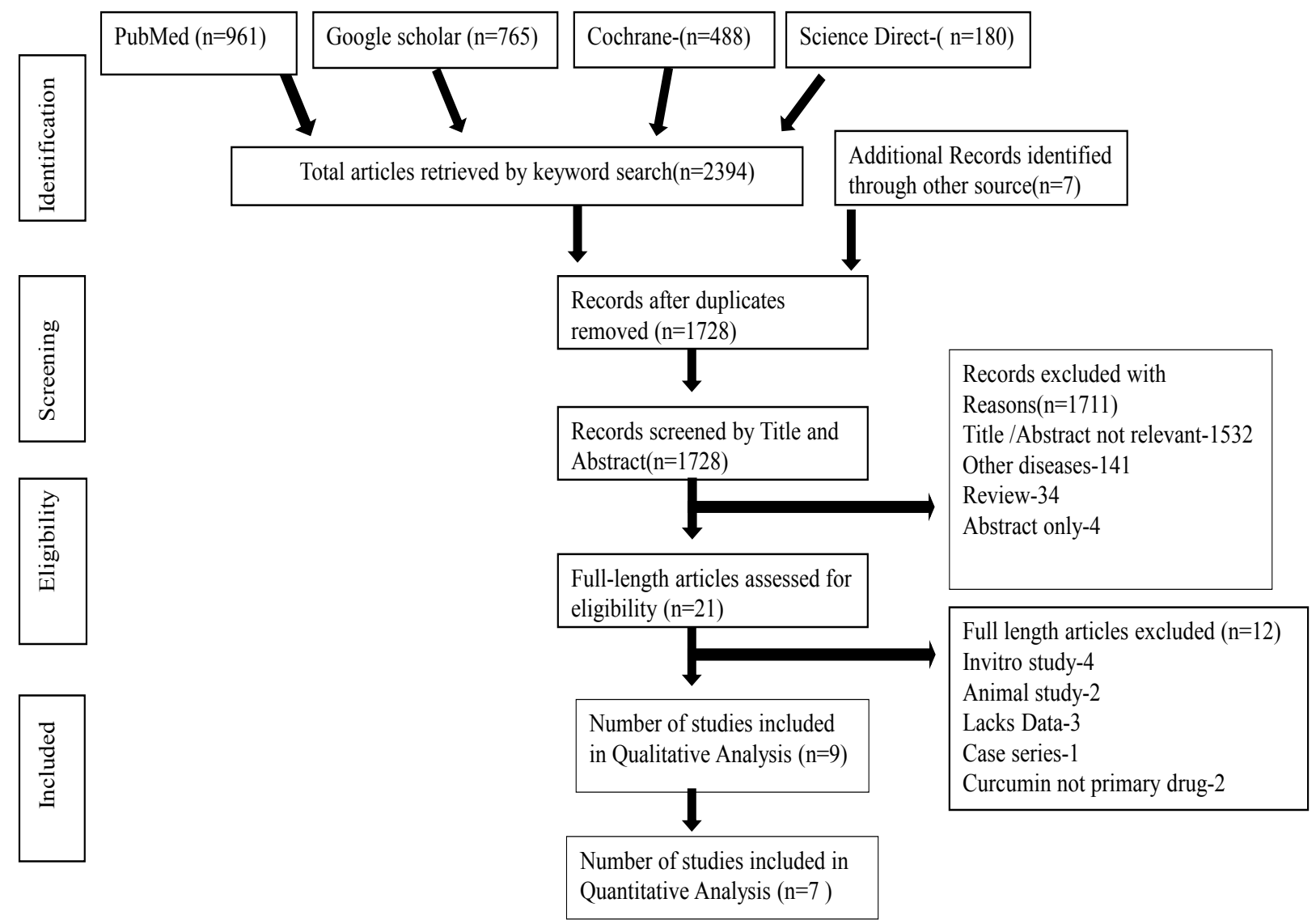

Figure 1. PRISMA Search Flow Chart Showing the Sources and the Final Short Listed Articles Included in This Review

days (Delavarian et al., 2019), 6 weeks (Rao et al., 2014), (Shah et al., 2021), 8 weeks (Mansourian et al., 2015), 2 months (Arun et al., 2020; Adhvaryu et al., 2018).

\section{Participants Characteristics}

The number of participants who took part in studies varied in size from 20-80 in number. Overall 582 patients with $\mathrm{HNC}$ undergoing RT/RCT were included in this systematic review. Male participants were 413, female participants were 129. 1 study didn't report gender (Charanthimath, 2016). In 6 studies patients underwent radio-chemotherapy (Rao et al., 2014; Patil et al., 2015; Charanthimath, 2016; Arun et al., 2020; Adhvaryu et al., 2018; Saldanha and Almeida., 2014), 3 studies radiotherapy (Mansourian et al., 2015, Delavarian et al.,2019; Shah et al., 2021). Regarding the age, two study

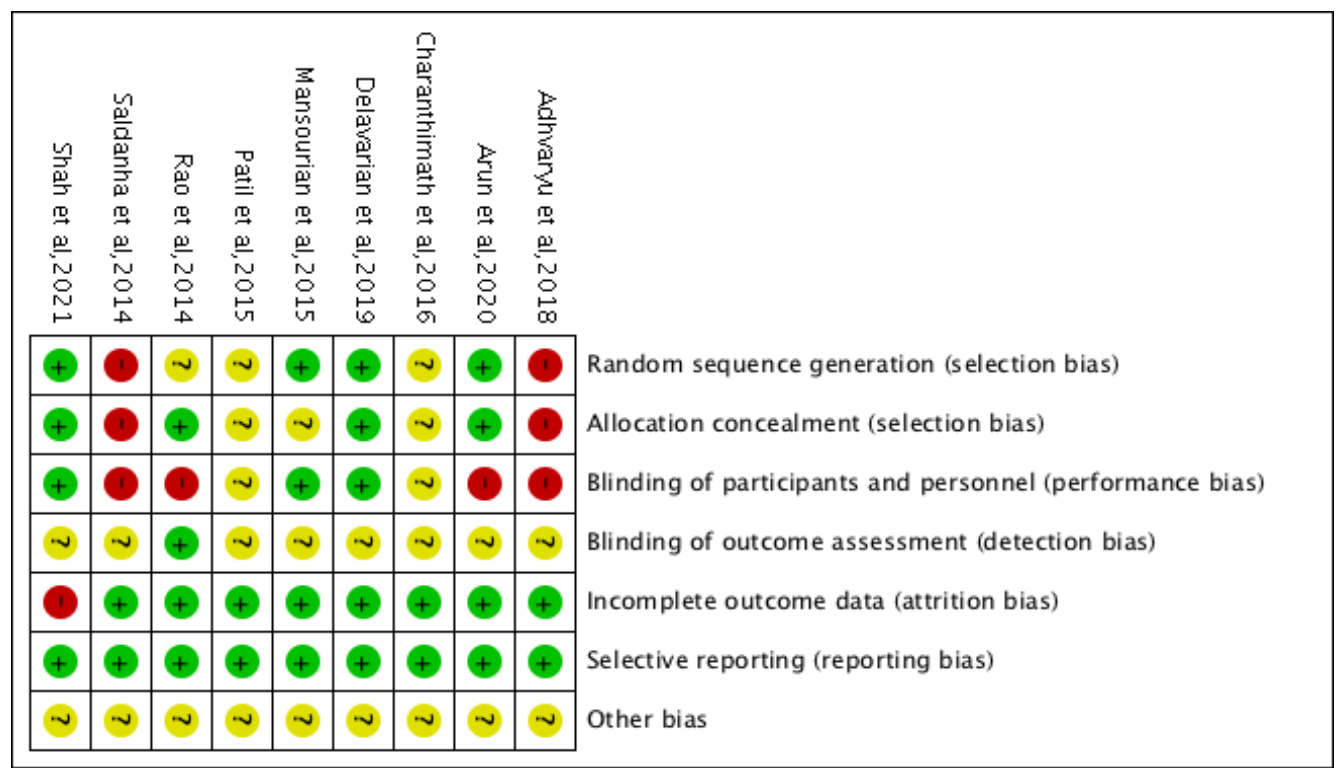

Figure 2. Risk of Bias and Applicability Concerns Summary: review authors judgements about each domain for each included study 


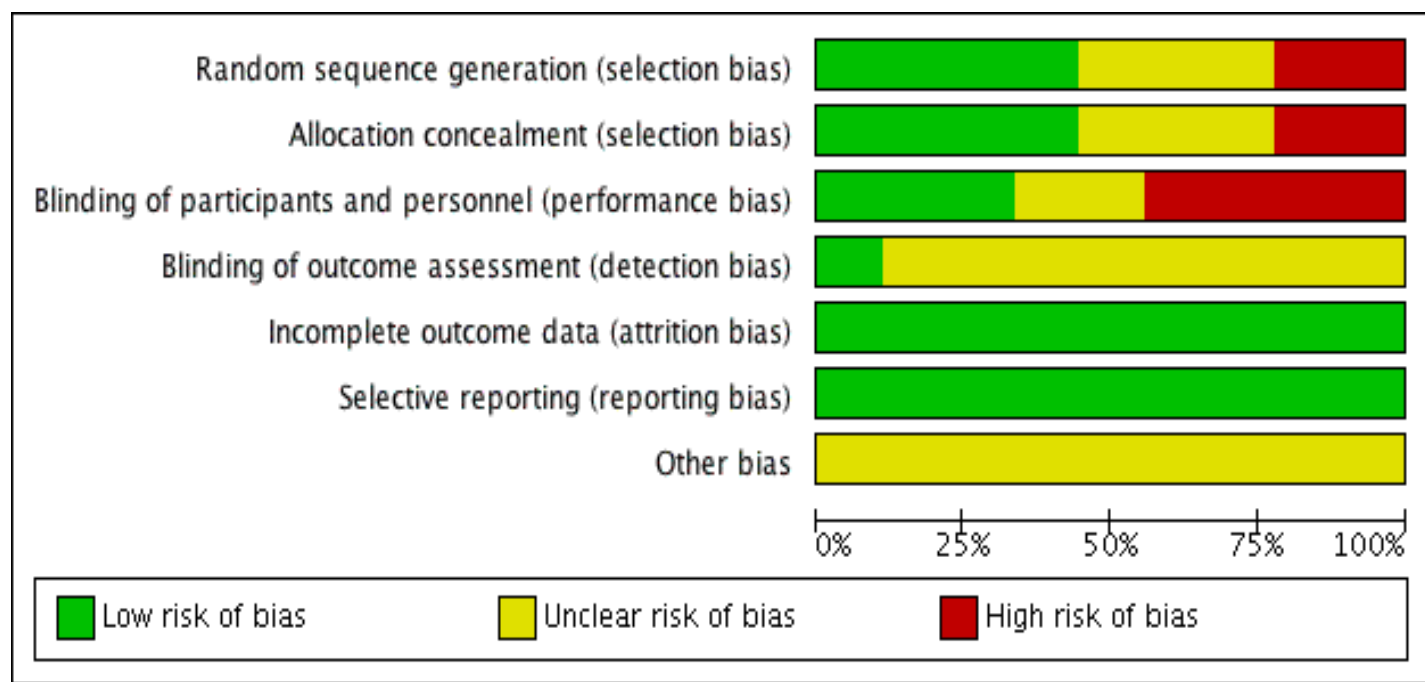

Figure 3. Risk of Bias and Applicability Concerns Graph: review authors' judgements about each domain presented as percentages across included studies

didn't report the age of the participants (Mansourian et al., 2015; Charanthimath, 2016), age range from 26-85 years (Rao et al., 2014), 39-70 years (Patil et al., 2015), 30-90 years (Arun et al., 2020), 10-90 years (Adhvaryu et al., 2018), 31-75 years (Saldanha and Almeida., 2014), 26-96 years (Shah et al., 2021), mean age 62.18+-15.07 (Delavarian et al., 2019). Table 1 shows Characteristics of Included Studies.

\section{Intervention Characteristics}

9 included studies evaluated the varied drug formulations of Curcumin. 2 clinical trials used Curcumin gel (Mansourian et al., 2015; Charanthimath, 2016). Curcumin mouthrinse $0.004 \%$ (Patil et al., 2015), $0.1 \%$ Curcumin nanoparticle $10 \mathrm{ml}$ mouthwash (Shah et al.,
2021), Turmeric gargle $10 \mathrm{ml}$ (Rao et al., 2014), Turmeric mouthwash (Saldanha and Almeida., 2014), capsule nanomicelle $80 \mathrm{mg}$ (Delavarian et al.,2019), turmeric extract capsule $500 \mathrm{mg}$ (Arun et al.,2020), oral $650 \mathrm{mg}$ Curcumin fortified with $13 \mathrm{mg}$ piperine (Adhvaryu et al., 2018). Interventions were prescribed 2 min 6 times a day(Rao et al.,2014),3 times/day for 7 days (Shah et al., 2021), 3 times a day gel (Mansourian et al., 2015),1:1,1 min thrice daily (Patil et al.,2015), 1 capsule/day orally (Delavarian et al,2016), 1 capsule thrice daily after food from first day of radiation (Arun et al,2020), 3 times a day 3 days prior to start of RT (Adhvaryu et al., 2018), thrice daily for 5 days Saldanha and Almeida., 2014), Patients had to avoid eating or drinking for 15 minutes before the initiation of radiotherapy, they had to cover

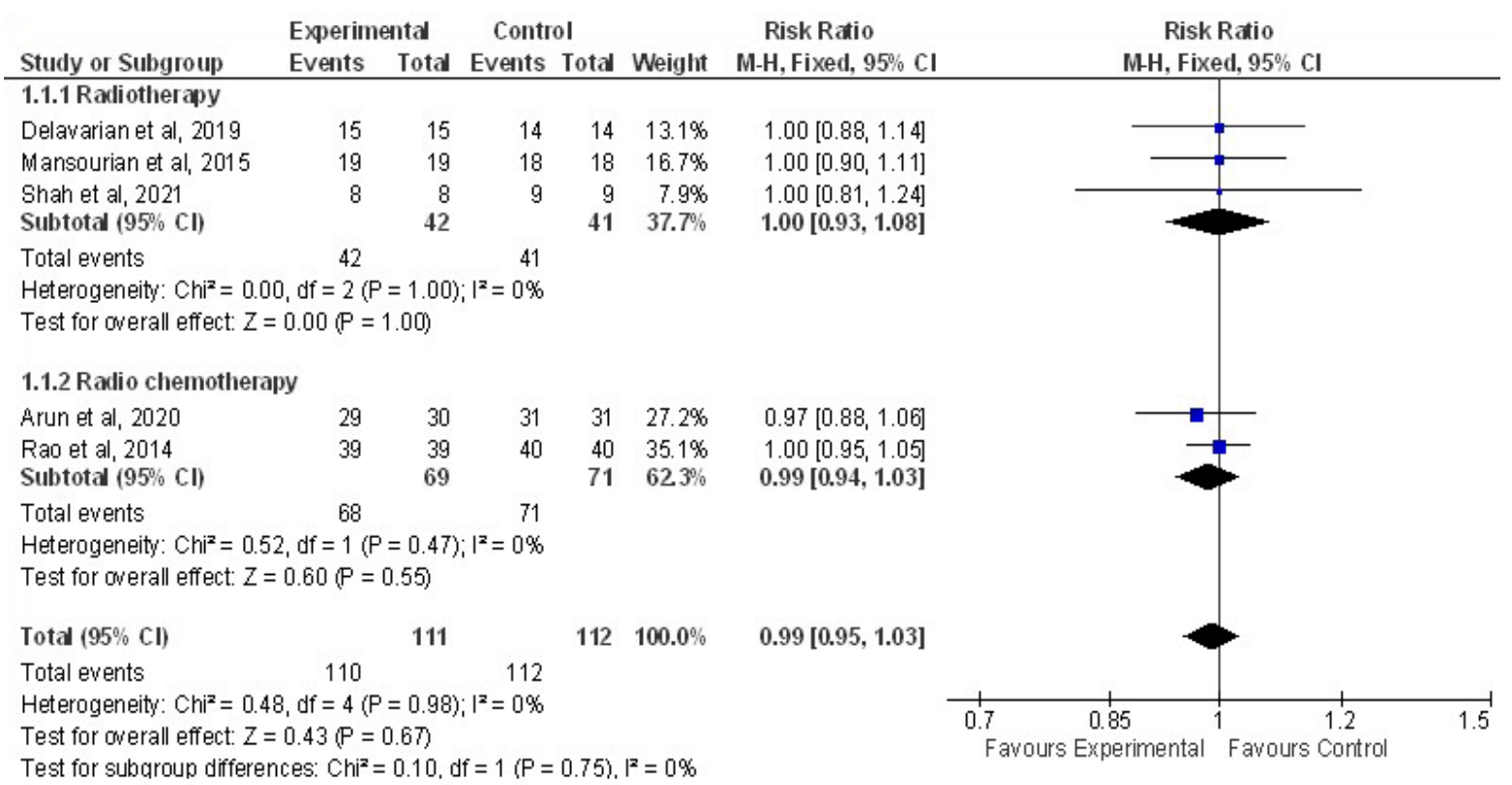

Figure 4. Forest Plot Depicting the Meta-Analysis Results of Curcumin Effect on Overall Incidence of Oral Mucositis Compared with Control in the Prophylactic Phase. Results plotted in left hand side indicate effect in favor of Curcumin and the combined effect including variance is plotted as black diamond at the bottom of forest plot. Analysis was done per subgroup (Radiotherapy and Radiochemotherapy). Events: Patients who had onset of OM. 


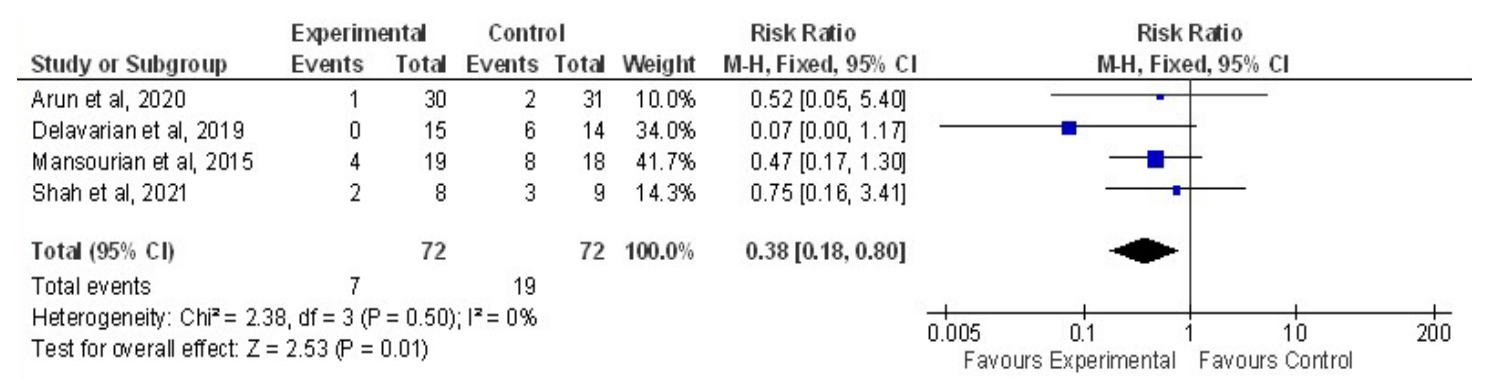

Figure 5. Forest Plot Depicting the Incidence of Delay in Onset of Oral Mucositis (Grade1, week 1) among Curcumin and Control Group

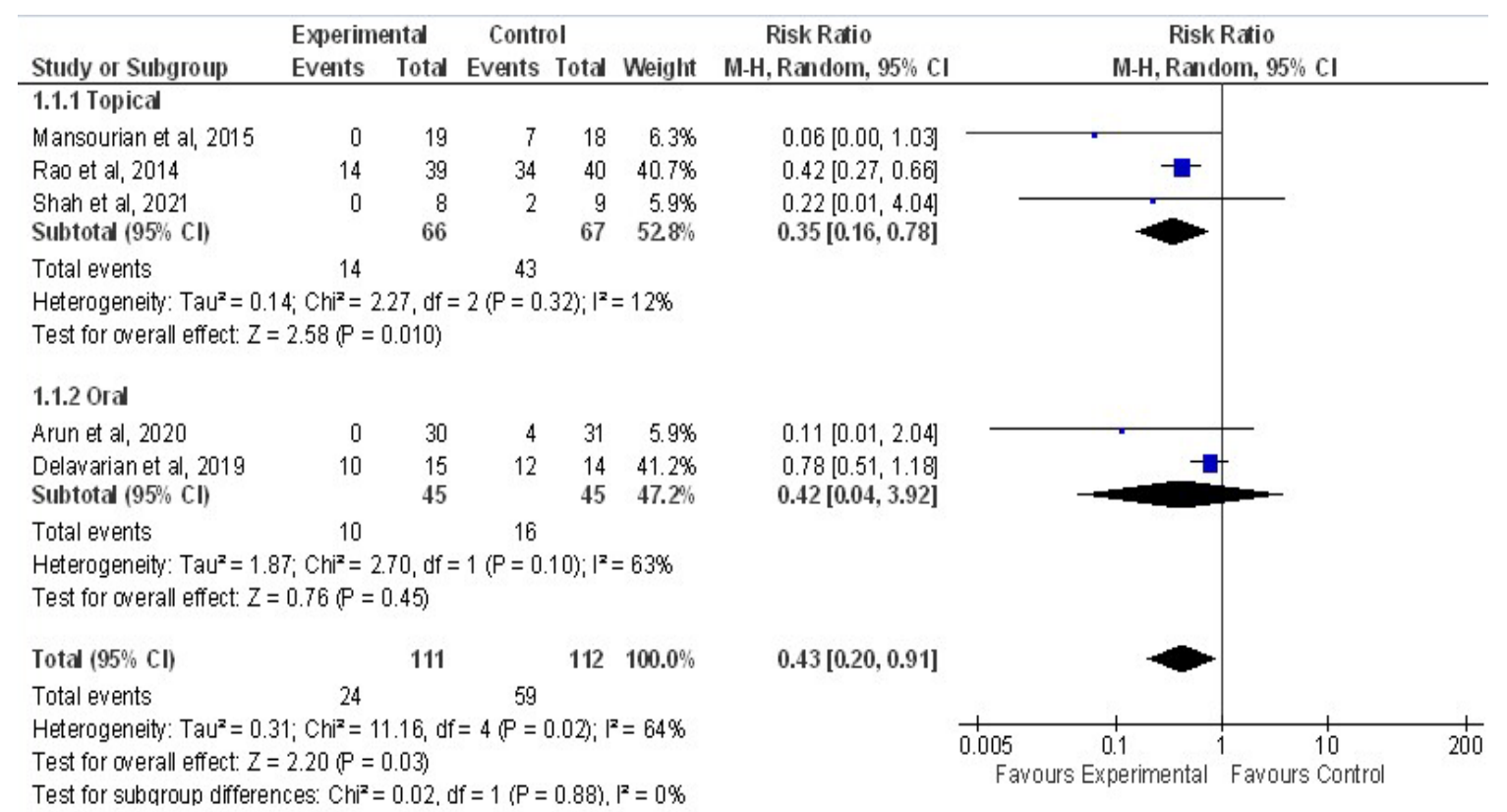

Figure 6. Forest Plot Depicting the Meta-Analysis Results of Curcumin Effect on Incidence of Severe ((Grade $>3)$ Equal or more to 3) Oral Mucositis Compared with Control in Prophylactic Phase. Results plotted in left hand side indicate effect in favor of Curcumin and the combined effect including variance is plotted as black diamond at the bottom of forest plot. Analysis was done per subgroup (Topical and Oral). Events: Patients who had OM severity with grade $>3$ more or equal to 3 after treatment.

whole mouth with a thin layer of gel by cotton applicator. Gel was applied for all radiotherapy duration 21 days 3 times a day (Mansourian et al., 2015). Variations were observed in control agents. Chlorhexidine gel (Charanthimath,2016), Placebo topical gel (Mansourian et al., 2015), Chlorhexidine mouthwash (Patil et al.,2015), Povidone Iodine (Rao et al.,2014), Placebo tablets-Lactose (Delavarian et al., 2019), placebo capsules Conventional radio-chemotherapy (Adhvaryu et al., 2018), Saline mouthwash (Saldanha and Almeida., 2014), 0.15\% benzydamine mouthwash (Shah et al., 2021).

\section{Clinical Parameters}

Grading of oral mucositis were assessed by OMAS in 3 studies (Patil et al., 2015), (Charanthimath et al., 2016), (Saldanha and Almeida., 2014), NRS in 2 studies (Patil et al.,2015; Charanthimath, 2016), VAS in 1 study (Mansourian et al., 2015), WHO Mucositis scale in 6 studies (Mansourian et al.,2015; Patil et al., 2015; Charanthimath, 2016; Arun et al., 2020; Adhvaryu et al.,

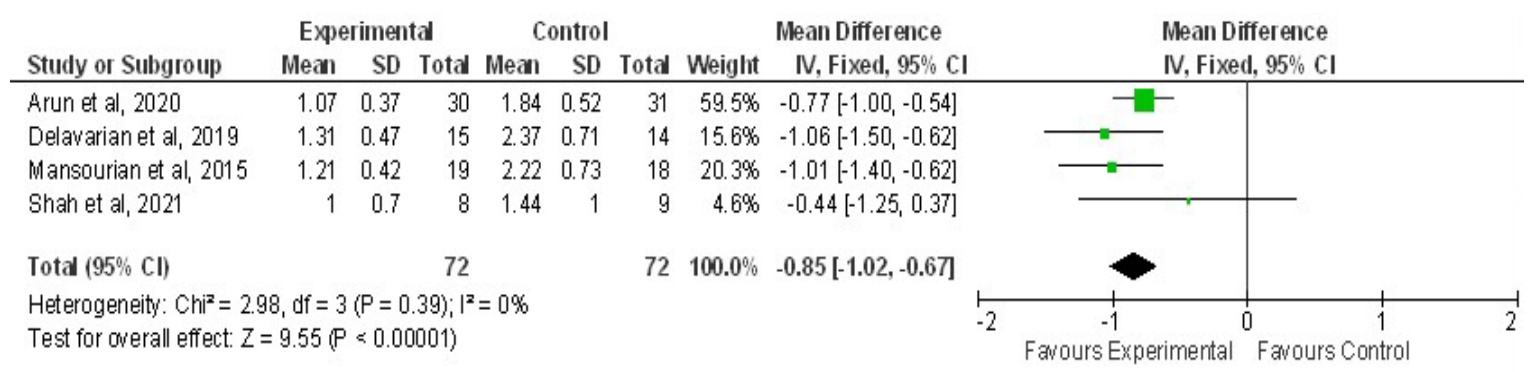

Figure 7. Forest Plot Depicting the Mean Mucositis Grading (Grade 3) among Curcumin and Control group 


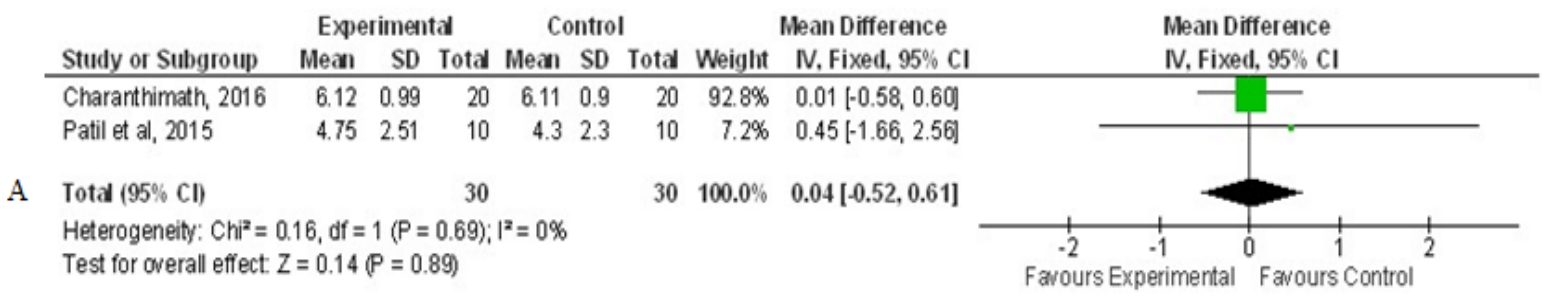

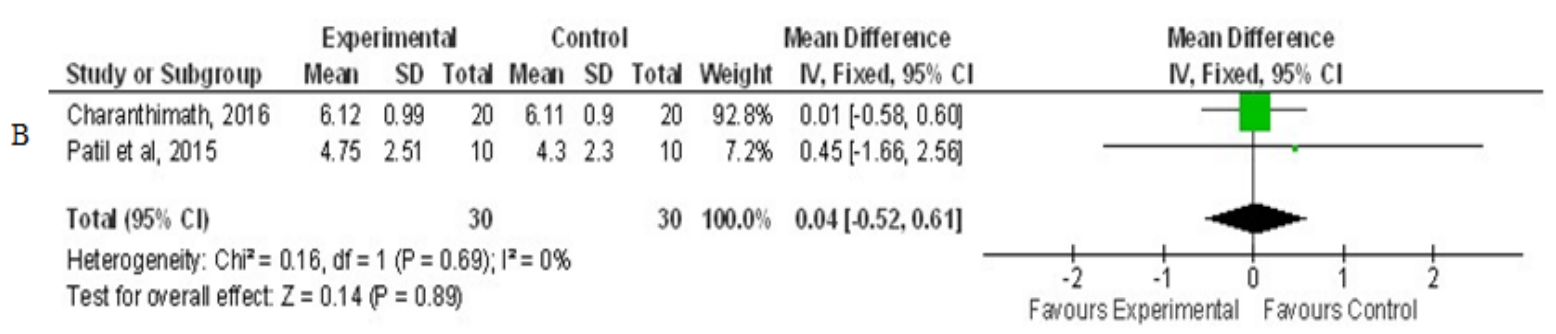

Figure 8. Forest Plot Depicting Pain Scores of Curcumin and Control in Therapeutic Phase. A, Baseline pain scores; B, Second Follow up

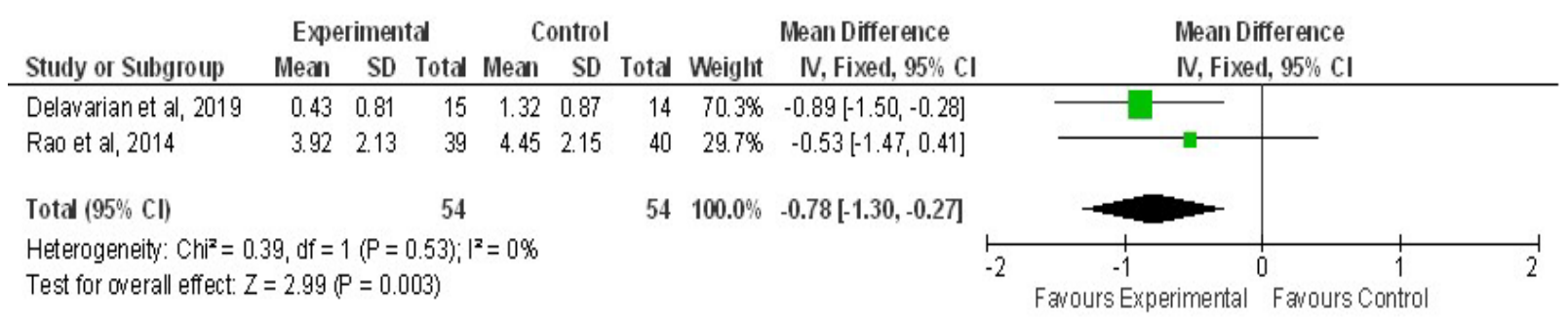

Figure 9. Forest Plot Depicting the Mean Change of Weight loss between Curcumin and Control Group.

2018; Shah et al., 2021), RTOG in 1 study (Rao et al., 2014), NCI-CTC v.2 in 1 study (Delavarian et al., 2019), NCI CTCAE assessment V.4 (Saldanha and Almeida., 2014)

\section{Risk of bias}

Among 9 studies, according to Cochrane Risk of Bias tool the estimated risk of bias was "low" in 3 studies (Mansourian et al., 2015; Delavarian et al., 2019; Shah et al.,2021), "moderate" in 6 studies (Rao et al.,2014; Patil et al., 2015; Charanthimath, 2016; Arun et al.,2020; Adhvaryu et al., 2018; Saldanha and Almeida., 2014). Only RCTs were assessed for randomization, allocation, blinding, rather non RCTs had either negative or unclear responses. Method used to generate sequence of randomisation was given only by 4 studies (Mansourian et al., 2015; Delavarian et al., 2019), Arun et al., 2018; Shah et al., 2021), balanced block randomization method using computer-generated random number table (Mansourian et al., 2015; Delavarian et al., 2019), 4x4 block randomisation sequence generated (Arun et al., 2020). Allocation concealment were done in 3 studies using opaque envelopes (Rao et al., 2014), identical containers (Delavarian et al., 2019), (Shah et al., 2021) sequentially numbered containers (Arun et al., 2020).1 RCT is triple blinded, parallel arm with intention to treat and Per protocol analysis done (Shah et al., 2021), 2 RCTs (Mansourian et al., 2015), Delavarian et al.,
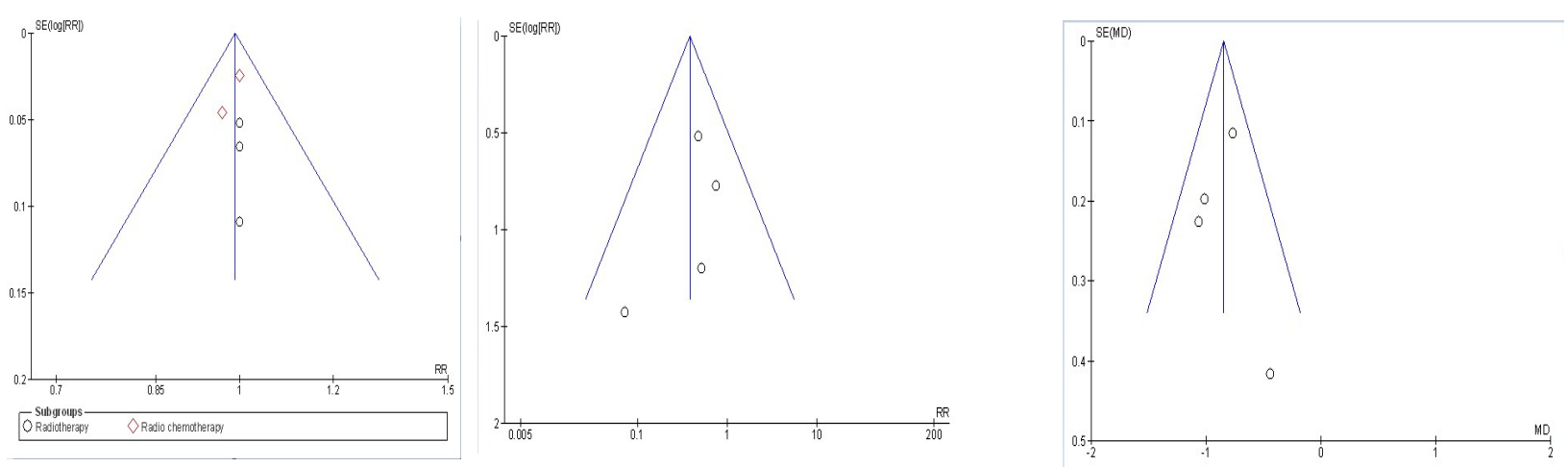

Figure 10. Funnel Plot Depicting Various Studies within Triangular Graph for the Analysis of Publication Bias in the Meta-Analysis for the Comparison of Curcumin and Control Group. A, Overall incidence of OM (5 Studies); B, Delay in onset of OM (4 studies); C. Mean Mucositis Grade-Grade 3 (4 studies) 
2019) were double blinded, one study had blinding of outcome assessor (Rao et al., 2014). 3 studies had patient withdrawal from study after randomisation (Rao et al., 2014; Delavarian et al., 2019; Shah et al., 2021). Loss to follow up were disclosed and prespecified outcome were reported in studies. All these were considered under low risk of bias. In Shah et al., (2021) there were large loss to follow up, but they were balanced across groups. 2 RCT was single blinded (Rao et al., 2014; Arun et al., 2020) which were considered as high risks. Description not provided due to missing and incomplete information were put under unclear risk. Majority of RCTs had not mentioned flow of participants details, intention to treat analysis, estimated effect size 95\%Confidence interval. Figures 2, 3 depicts risk of bias summary and graph for review of authors' judgements about each domain for the included studies.

\section{Study Description}

Effect on Onset of Oral Mucositis

6 studies (Rao et al., 2014; Delavarian et al., 2019; Arun et al., 2020; Adhvaryu et al., 2018; Shah et al., 2021; Mansourian et al., 2015) assessed the curcumin/turmeric effects on the onset of OM. Delavarian et al., (2019) showed that there was delay in onset of $\mathrm{OM}$ in nanocurcumin group compared to control group $(\mathrm{P}=0.002)$, only $25 \%$ developed grade $1 \mathrm{OM}$ in $2^{\text {nd }}$ week. Arun et al., (2020) reported that patients in turmeric group showed decrease in incidence of OM compared to placebo after 3 weeks of treatment. $86.7 \%$ of patients had grade $1 \mathrm{OM}$ in turmeric group whereas $71 \%$ had grade $2 \mathrm{OM}$ in placebo group. A study by Adhvaryu et al., (2018) observed significant decrease in incidence of OM from $92 \%$ to $51 \%(\mathrm{P} \leq 0.001)$ among control and curcumin treated group was observed. Onset of tolerable and intolerable mucositis was delayed in the patients using turmeric showed statistically significant difference compared to controls in a study by Suresh Rao et al., (2014). Shah et al.,2021 showed that nine (100\%) patients in benzydamine group and six (75\%) patients in curcumin group experienced OM .

\section{Effect on Degree of severity}

A total of 6 studies (Rao et al.,2014, Mansourian et al.,2015, Delavarian et al.,2019, Arun et al.,2020, Adhvaryu et al.,2018, Shah et al.,2021) assessed the curcumin/turmeric effects on degree of severity. The study by Mansourian et al., (2015) found that there was no grade 3 mucositis, time the symptom started was longer, mean size of oral lesion, erythema, burning sensation in curcumin group was significantly lower than control group $(p<0.0001)$. The study by Rao et al., (2014) reported that turmeric showed statistical significance of $(p<0.0001)$, 14 of 39 patients with intolerable mucositis whereas in povidone-iodine group 34 of 40 patients. In the RCT by Delavarian et al., (2019) 50\% of grade 3 OM was observed after 3 weeks in the control group whereas only $33 \%$ of study group reached this grade after 4 weeks which was statistically significant $(\mathrm{P}<0.05)$. Similarly, Arun et al., (2020) also showed in turmeric treated group none showed grade $3 \mathrm{OM}$ and $73.3 \%$ had grade 1 after 4 th week of treatment whereas in control group 13\% showed grade 3 OM and 68\% had grade 2. Adhvaryu et al.,(2018) observed significant decrease in grade III and IV mucositis from $51.6 \%$ to $12.8 \%(\mathrm{P} \leq 0.001)$ among control and curcumin treated group respectively. Shah et al,(2021) showed that both mouthwashes curcumin and Benzydamine were equally effective in preventing the occurrence of severe form of oral mucositis.

\section{Grading of Oral Mucositis $O M A S$}

Patients clinically diagnosed with oral mucositis managed with Curcumin/Turmeric in 3 studies (Patil et al., 2015; Charanthimath, 2016; Saldanha and Almeida 2014) used OMAS for grading. 1 study by Saldanha and Almeida (2014) compared score of Treatment Induced Oral Mucositis in pre and post test. The difference between the test were more in turmeric group compared to saline mouth wash proving that turmeric mouth wash was better than saline mouth wash. 1 study by Charanthimath (2016) observed percentage of change in erythema and size of ulcer from minimal changes to total reduction, significant difference was obtained from baseline, first and second week follow ups and another study by Patil et al., (2015) showed erythematous and ulceration scores were statistically significant $\mathrm{p}=0.050$ and $\mathrm{p}<0.001$ from baseline to second follow up.

\section{WHO Mucositis Score}

WHO Mucositis score were measured in 5 studies (Mansourian et al., 2015; Patil et al., 2015; Charanthimath, 2016; Arun et al., 2020; Adhvaryu et al., 2018; Shah et al., 2021). RCT of Charantimath (2016), observed change in erythema and ulcer healing and showed improvement in first and second week which were statistically significant in curcumin group. A Study by Patil et al., (2015) reported that WHO Scores $(p=0.003)$ were statistically significant from baseline to second follow up.1 study by Adhvaryu et al., (2018) showed a significant reduction in incidence of oral mucositis and in grade III and IV mucositis in curcumin treated group.1 study by Arun et al., (2020) majority of the patients had grade 1 mucositis and none had grade 3 mucositis in turmeric group compared to placebo where majority had grade 2 and few had grade 3 mucositis at the end of fourth week. Study by Shah et al.,2021 majority had developed grade 1 and 2, none had grade 3 mucositis in curcumin group when compared to Benzydamine.

\section{RTOG Grading}

1 study by Rao et al., (2014) assessed OM using RTOG grading system showed turmeric group had delayed and reduced intolerable mucositis $(\mathrm{P}<0.001)$.

\section{NCI CTC}

The study by Delavarian et al., (2019) had assessed grading of OM by NCI CTC v.2, only $32 \%$ of curcumin group developed OM in 2nd week of radiotherapy whereas all in control group. Subjective scale NCI CTCAE were used to assess OM in study by (Arun et el.,2020) 


\section{Pain Assessment}

In two clinical trials comparison between curcumin and chlorhexidine, NRS score showed reduction in pain ( $\mathrm{p}$ value $=0.0001)$ in a study by Charanthimath (2016) and scores were better from baseline till 2nd follow $u p(p<0.001)$ in curcumin group in another study by Patil et al., (2015). VAS analysis showed reduction in burning sensation in topical curcumin group compared to placebo group (Mansourian et al., 2015).

\section{Treatment breaks}

Rao et al., (2014) had reported that $17.9 \%$ had treatment delay at $6 \mathrm{th} / 7$ th week of radiation, whereas in Povidone iodine it was $24 \% .1$ study by Adhvaryu et al., (2018) showed that patient completing radiotherapy dose schedule increased in curcumin group of $80 \%$ indicating the reduced treatment breaks.

\section{Treatment days lost}

The study by Rao et al., (2014) observed the days lost during treatment were not statistically significant between povidone iodine and turmeric as it was $7.25-0.56$ and 7 - 0 days respectively.

\section{Loss of Body Weight}

The study by Delavarian et al., (2019) showed average loss of body weight as $0.43 \pm 0.83 \mathrm{~kg}$ in nanomicelle curcumin group compared to control group was $1.32 \pm$ $0.87(\mathrm{P}=0.003)$. 1 study by Rao et al., (2014) reported weight loss was statistically significant more in Povidone iodine group which was $4.45 \pm 2.15$, compared to turmeric group which was $3.92 \pm 2.13(\mathrm{P}<0.001)$.

\section{Quantitative Synthesis}

Meta-analysis was performed based on 7 articles with total 283 patients with 141 cases and 142 controls were included. Pooling of five trials were done to assess the effect of curcumin on overall incidence of OM compared to controls. As shown in Figure 4, the random effects model was used and showed that results were not statistically significant $(\mathrm{Z}=0.43, \mathrm{P}=0.67)$ between curcumin and control group in preventing onset of OM with an overall effect size of 0.99 at $95 \% \mathrm{CI}=0.95,1.03$. No heterogeneity of included studies were found. $\mathrm{Chi} 2=0.10, \mathrm{df}=1(\mathrm{P}=0.75) \mathrm{I}^{2}=0 \%$ Analysis was done per subgroup (Radiotherapy and Radiochemotherapy).Studies done in Radiotherapy (three studies with 83 patients, $\mathrm{I}^{2}=0 \%$ ) and in Radiochemotherapy (two studies with 140 patients, $\mathrm{I}^{2}=0 \%$. In studies with Radio-chemotherapy with RR of 0.99 at $(95 \% \mathrm{CI}=0.94,1.03)$ and in Radiotherapy with RR of 1.00 at $(95 \% \mathrm{CI}=0.93,1.08)$, irrespective of cancer treatment given curcumin were not effective in preventing overall incidence of OM.

Pooling of five RCTs were done to assess the effect of curcumin on severity of OM (Grade more or less equal 3) compared to controls is shown in Figure 6. In curcumin group, with 111 patients only 24 had developed severe OM, in control group among 112 patients 95 developed severe OM. Result showed an overall effect size, RR of $0.43(95 \% \mathrm{CI}=0.20,0.91)$. Result were statistically significant between curcumin and control group $\mathrm{Z}=2.20(\mathrm{P}=0.03)$ in reducing severity of $\mathrm{OM}$. As heterogeneity of included studies were $\mathrm{Chi} 2=11.16, \mathrm{df}=4$ $(\mathrm{P}=0.02) \mathrm{I}^{2}=64 \%$, subgroup analysis were done with Topical and oral curcumin. Studies done in topical (three studies with 133 patients, $\mathrm{I}^{2}=12 \%$ ) and in Oral (two studies with 90 patients, $\mathrm{I}^{2}=63 \%$. There was a significant preventive effect of topical curcumin in reducing severity of OM with RR of 0.35 at $(95 \% \mathrm{CI}=0.16,0.78)$ compared to oral with $\mathrm{RR}$ of 0.42 at $(95 \% \mathrm{CI}=0.04,3.92)$.

Four Trials were pooled to assess the effect of curcumin on delay in onset of OM compared to controls is shown in Figure 5. Among 72 patients only 7 developed grade $1 \mathrm{OM}$ in curcumin group whereas 19 developed in control group in week 1 . Results are statistically significant $(\mathrm{Z}=2.53, \mathrm{P}=0.01$ ) between curcumin and control group in delaying the onset of $\mathrm{OM}$ with an overall effect size of 0.38 at $95 \% \mathrm{CI}=0.18,0.80$. No heterogeneity of included studies were found. Chi $2=2.38, \mathrm{df}=3(\mathrm{P}=0.50) \mathrm{I}^{2}=0 \%$

Figure 7 showed pooling of 4 RCTs were done to evaluate the mean difference for OM scores at grade 3 mucositis. OM mean severity reduced with curcumin usage when compared to control, statistically significant $(Z=9.55, P<0.00001)$ with overall effect of -0.85 at $95 \% \mathrm{CI}=-1.02,0.67)$. No heterogeneity of included studies were observed, Chi2 $=2.98, \mathrm{df}=3(\mathrm{P}=0.39), \mathrm{I}^{2}=0 \%$. Figure 8 showed combined analysis of two trials of pain intensity from NRS score revealed mean difference of pain reduction with overall effect of -2.17 at $95 \% \mathrm{CI}(-2.77,-1.58)$ was more in curcumin group when compared to chlorhexidine group statistically significant, $\mathrm{Z}=7.15(\mathrm{P}<0.00001)$, with heterogeneity Chi $2=1.18, \mathrm{df}=1(\mathrm{P}=0.28), \mathrm{I}^{2}=15 \%$. Combined analysis of two trials of mean change of weight between curcumin and control revealed mean difference with overall effect of -0.78 at $(95 \% \mathrm{CI}=-1.30,-0.27)$ was effective in preventing weight loss in curcumin group when compared to control group statistically significant, $\mathrm{Z}=2.99(\mathrm{P}=0.003)$, with heterogeneity $\mathrm{Chi} 2=0.39$, $\mathrm{df}=1(\mathrm{P}=0.53), \mathrm{I}^{2}=0 \%$ as shown in Figure 9.

\section{Publication Bias}

Figure 10 showed funnel plots indicating publication bias within triangular graph included in the meta-analysis for the comparison of Curcumin and control group. A. indicates no evidence of publication bias among 5 studies identifying overall incidence of OM with subgroup radiotherapy and radiochemotherapy. In B. Delay in onset of OM and C. Mean OM Grading (Grade 3), each included 4 studies showed 1 study is dispersed down might be due to an included smaller trial indicating publication bias.

Adverse effect: From 9 articles only 2 studies reported adverse effect. Mansourian et al., (2015) reported nausea after using the gel, which got resolved after 2 weeks. Adhvaryu et al., (2018) reported mild stomach upset in $10 \%$ of cases.

\section{Discussion}

Most debilitating inevitable dose limiting toxicity of oral cancer therapy such as radiotherapy and chemotherapy is oral mucositis (Rodriguez et al., 2012). Quality of life in these patients are hampered due to its severe complications 
(Mercadante et al., 2015).

The present meta-analysis included 7 trials finding the efficacy of curcumin in treatment of oral mucositis in patients undergoing radiotherapy, radiochemotherapy for head and neck cancer. The prophylactic use of curcumin effectively delayed the incidence of onset with RR of 0.38 and reduced the severity of oral mucositis with RR of 0.48 , statistically significant respectively compared to controls but there was no effectiveness in prevention of the overall incidence of oral mucositis with RR of 0.99 , not statistically significant. There was moderate heterogeneity $64 \%$ among topical and oral administration due to methodological difference included in formulation and dosages. Oral mucositis mean severity reduced with curcumin usage when compared to control with overall mean difference of- 0.85 , statistically significant. Prevention of Reduction in weight loss was effective in curcumin over controls with MD of -0.78 , statistically significant.

Cancer treatment induced mucositis cause acute pain due to inflammation, sloughing of tissue and ulcerations. (Lalla et al.,2014). These are subjective measures that has effect in its clinical management. VAS, NRS are reliable and valid pain tool. Therapeutic use of curcumin effectively reduced pain scores graded using NRS in already developed OM with MD of -2.17 , statistically significant.

Topical therapies are more easily applied, not expensive and has lesser adverse effects compared to systemic therapies. (Ana et al.,2020). Topical curcuma longa gel prepared by using $500 \mathrm{gm}$ of fresh curcumin powder effectively decreased oral symptoms by reducing the size of oral lesions, improving the grades of oral mucositis in HNC patients undergoing radiotherapy (Mansourian et al.,2015). In other study, commercially available Curcumin gel was compared with chlorhexidine gel that had faster healing of wound that was safer and an effective alternative (Charanthimath, 2016). Use of $0.1 \%$ of nanocurcumin mouthwash delayed the onset of Oral mucositis (Shah et al.,2021). Curcumin mouthwash $0.004 \%$ was better tolerated and effective in reducing the signs and symptoms of chemo-radiotherapy induced oral mucositis (Patil et al.,2015). Turmeric gargle prepared from 400mg turmeric capsule reduced and delayed the severity of OM. They had less treatment breaks and favoured food intake due to reduced mucositis. Frequency of curcumin formulations used thrice to six times showed improvement in oral mucositis and reduced size of oral lesions. (Rao et al.,2014). Saldanha et al.,2014 stated that turmeric mouthwash prepared from $1500 \mathrm{mg}$ turmeric powder was better than saline mouthwash. Methodology of formulary preparation for topical turmeric/curcumin among studies varied, hence requires standardised formulation with more RCTs. There was a significant preventive effect of topical curcumin in reducing severity of OM with RR of 0.35 compared to oral with RR of 0.42 .

Curcumin has limited bioavailability, poor absorption, metabolised and excreted rapidly when consumed orally. To combat this, Delavarian et al., (2019) in their study considered oral curcumin nanomicelle $80 \mathrm{mg} /$ day tablet as an effective and safe agent in preventing the development and reducing the severity of OM, it also has the potency to lower weight loss. Curcumin entrapped nanoparticles produced enhanced oral bioavailability, curcumin solubility in aqueous solutions and absorption achieving the considerable serum and tissue levels and uptake by different tissues (Shaikh et al.,2009). Nanoparticles of curcumin are miscible in water. (Shah et al.,2021). Adhvaryu et al., (2018) suggested oral curcumin to nearly $2000 \mathrm{mg} /$ day fortified with piperine enhanced its bioavailability, reducing the incidence of grade III and IV mucositis, reduced drop-out rate with improved patient compliance. Arun et al.,(2020) reported bioavailable turmeric extract $1500 \mathrm{mg} /$ day capsule after food reduced the incidence and severity of OM with no systemic toxicity and was safe to use.

In a recent study by Shah et al., (2021) Modified Intention to treat (MIT) had sample size $n=35$ in Benzydamine group and $n=33$ in curcumin group initially, due to large loss to follow up reduced to $\mathrm{n}=9$ in Benzydamine and $\mathrm{n}=8$ in curcumin group. Per protocol(PP) analysis excluded patients who deviated from protocol, but lacked similarity in characteristics can introduce attrition bias. Results may provide low level of evidence due to small sample size but certainly will reflect the reliable estimation of treatment effects. MIT analysis was not taken into consideration as it may overestimate the effect.

Among six preventive studies (Rao et al.,2014; Mansourian et al., 2015; Delavarian et al., 2019; Arun et al.,2020; Adhvaryu et al., 2018; Shah et al., 2021) using turmeric/curcumin formulations, there were significant benefits than comparator were better in delaying the onset of OM, showed reduction in severity of OM, three (Rao et al., 2014; Delavarian et al., 2019; Adhvaryu et al., 2018) studies found reduction in treatment breaks, weight loss, hence supporting the effectiveness of turmeric/ curcumin in treatment of OM. Three studies (Rao et al., 2014; Mansourian et al., 2015; Shah et al., 2021) were topical and three (Delavarian et al., 2019; Arun et al., 2020; Adhvaryu et al., 2018) were oral. Three therapeutic studies (Patil et al., 2015; Charanthimath, 2016; Saldanha and Almeida., 2014) using topical Curcumin/Turmeric showed reduction in Pain, erythema, ulcer size when compared to controls. Two studies (Patil et al., 2015; Charantimath, 2016) with topical curcumin in comparison with chlorhexidine was efficacious, safer and was better in rapid wound healing and better patient compliance. Regular turmeric swish 6 times per day increasing the frequency was effective in preventing oral mucositis (Rao et al., 2014). 9 included studies revealed that turmeric/ curcumin as gel, mouthwash, orally was well tolerated, safe and are widely accepted.

From the above 9 clinical trials, Curcumin/Turmeric is effective in controlling signs and symptoms of radiochemotherapy induced OM in HNC patients with minimal adverse effects. They delay the onset and reduce severity of OM, with less drop-outs, reduced loss of body weight, reduced treatment days. New innovative Curcumin/ Turmeric formulations are needed that increase its bioavailability and better effectiveness.

Limitations were though there was difference in 
cancer treatment, curcumin/turmeric formulations, dosage, concentration, directions, therapy duration, controls, grading scales, follow ups across studies, all 7 trials with only 283 participants, studies published only in English had to be included in this analysis. Due to small sample size and limited randomised controlled trials included, they could have resulted in varying outcome. Overall methodological quality among studies were moderate.

In Conclusion, Curcumin/Turmeric are efficacious, well tolerated and safe in prevention and amelioration of $\mathrm{RT} / \mathrm{RCT}$ induced oral mucositis in HNC patients. There is moderate to strong evidence that curcumin is effective in delaying the onset and in reducing the severity of OM, mean mucositis severity, pain intensity, weight loss. Dosage required orally was less than $2,000 \mathrm{mg}$ /day of Curcumin/ Turmeric, $80 \mathrm{mg} /$ day $/ 0.1 \%$ mouthwash of nanocurcumin, topically gel/mouthwash used with increase in frequency/ day prior and during(prophylactic) and after(therapeutic) RT/RCT are beneficial with no noticeable side effects and are cost effective. However, multi-centred quality randomised controlled trials with innovative curcumin/ turmeric formulations are needed to further support the evidence in preventing and treating oral mucositis.

\section{References}

Adhvaryu M, Vakharia B, Reddy N (2018). Curcumin Prevents Mucositis and Improves Patient Compliance in Head \& Neck Cancer Patients Undergoing Radio-Chemotherapy. Ann Med Chem Res, 4, 1022.

Ana GS, Normando AGC, de Toledo IP, et al (2020). Topical treatment of oral mucositis in câncer patients: A systematic review of randomized clinical trials. Asian Pac J Cancer Prev, 21, 1851.

Anderson PM, Lalla RV (2020). Glutamine for Amelioration of Radiation and Chemotherapy Associated Mucositis during Cancer Therapy. Nutrients, 12, 1675.

Arun P, Sagayaraj A, Mohiyuddin SA, et al (2020). Role of turmeric extract in minimising mucositis in patients receiving radiotherapy for head and neck squamous cell cancer: a randomised, placebo-controlled trial. J Laryngol Otol, 134, 159- 4.

Bensinger W, Schubert M, Ang KK, et al (2008). NCCN task force report: prevention and management of mucositis in cancer care. J Nat Comprehensive Cancer Network, 6, S-1.

Bowen J, Al-Dasooqi N, Bossi P, et al (2019). The pathogenesis of mucositis: updated perspectives and emerging targets. Supportive Care Cancer, 27, 4023-3.

Campos TM, Silva CADPT, Sobral APT, et al (2020). Photobiomodulation in oral mucositis in patients with head and neck cancer: a systematic review and meta-analysis followed by a cost-effectiveness analysis. Supportive Care Cancer, 2020, 1-11.

Cardona A, Balouch A, Abdul MM ,et al (2017). Efficacy of chlorhexidine for the prevention and treatment of oral mucositis in cancer patients: a systematic review with meta-analyses. J Oral Pathol Med, 46, 680-8.

Charantimath S (2016). Use of curcumin in radiochemotherapy induced oral mucositis patients: A control trial study. Int $J$ Med Health Sci, 10, 147-2.

Correa MEP, Cheng, KKF, Chiang K, et al (2019). Systematic review of oral cryotherapy for the management of oral mucositis in cancer patients and clinical practice guidelines. Supportive Care Cancer, 2019, 1-8.

Delavarian Z, Pakfetrat A, Ghazi A ,et al (2019). Oral administration of nanomicelle curcumin in the prevention of radiotherapy-induced mucositis in head and neck cancers. Special Care Dentistry, 39, 166-2.

de Lima VHS, de Oliveira-Neto OB, da Hora Sales PH, et al (2020). Effectiveness of low-level laser therapy for oral mucositis prevention in patients undergoing chemoradiotherapy for the treatment of head and neck cancer: A systematic review and meta-analysis. Oral Oncol, 102, 104524.

Duncan GG, Epstein JB, Tu D, et al (2005). Quality of life, mucositis, and xerostomia from radiotherapy for head and neck cancers: a report from the NCIC CTG HN2 randomized trial of an antimicrobial lozenge to prevent mucositis. Head \& Neck: Journal for the Sciences and Specialties of the Head and Neck, 27, pp 421-8.

Epstein JB, Miaskowski C(2019). Oral pain in the cancer patient. JNCI Monogr, 2019, 45-53.

Elting LS, Keefe DM, Sonis ST, Burden of Illness Head and Neck Writing Committee (2008). Patient-reported measurements of oral mucositis in head and neck cancer patients treated with radiotherapy with or without chemotherapy: demonstration of increased frequency, severity, resistance to palliation, and impact on quality of life. Cancer, 113, 2704-3.

Hong CH, Gueiros LA, Fulton JS, et al (2019). Systematic review of basic oral care for the management of oral mucositis in cancer patients and clinical practice guidelines. Supportive Care Cancer, 27, 3949-7.

Khanal B, Baliga M, Uppal N (2010). Effect of topical honey on limitation of radiation-induced oral mucositis: an intervention study. Int J Oral Maxillofacial Surg, 39, 1181-5.

Lalla, RV, Bowen J, Barasch A (2014). MASCC/ISOO clinical practice guidelines for the management of mucositis secondary to cancer therapy. Cancer, 120, 1453- 1.

Logan R M, Al-Azri, AR, Bossi P, et al (2020). Systematic review of growth factors and cytokines for the management of oral mucositis in cancer patients and clinical practice guidelines. Supportive Care Cancer, 28, 2485-8.

Mansourian A, Amanlou M, Shirazian S, et al (2015). The effect of "Curcuma Longa" topical gel on radiation-induced oral mucositis in patients with head and neck cancer. Int J Radiat Res, 13, 269-4.

Mazieiro R, Frizon RR, Barbalho SM, et al (2018). Is curcumin a possibility to treat inflammatory bowel diseases?. J Med Food, 21, 1077- 5.

Mercadante S, Aielli F, Adile C (2015). Prevalence of oral mucositis, dry mouth, and dysphagia in advanced cancer patients. Supportive Care Cancer, 23, 3249- 5.

Mohanty C, Sahoo SK (2017). Curcumin and its topical formulations for wound healing applications. Drug Discovery Today, 22, 1582-92.

Murphy BA, Ridner S, Wells N (2007). Quality of life research in head and neck cancer: a review of the current state of the science. Crit Rev Oncol Hematol, 62, 251-7.

Nagpal M, Sood S (2013). Role of curcumin in systemic and oral health: An overview. J Nat Sci Biol Med, 4, 3 .

Nooka AK, Johnson HR, Kaufman JL, et al (2014). Pharmacoeconomic analysis of palifermin to prevent mucositis among patients undergoing autologous hematopoietic stem cell transplantation. Biol Blood Marrow Transplantation, 20, 852-7.

Normando AGC, de Menêses AG, de Toledo IP, et al (2019). Effects of turmeric and curcumin on oral mucositis: A systematic review. Phytotherapy Res, 33, 1318-9.

Nosratzehi T, Arbabi-Kalati F, Hamishehkar H, et al (2018). Comparison of the effects of curcumin mucoadhesive paste and local corticosteroid on the treatment of erosive oral lichen planus lesions. J Nat Med Assoc, 110, 92-7.

Asian Pacific Journal of Cancer Prevention, Vol 221683 
Paiar F, Cristaudo A, Gonnelli A, et al (2020). Radiation-induced nausea and vomiting in head and neck cancer: Is it something worth considering in the intensity modulated radiotherapy era?"A narrative review”. Head Neck, 42, 131-7.

Patil K, Guledgud MV, Kulkarni PK, et al (2015). Use of curcumin mouthrinse in radio-chemotherapy induced oral mucositis patients: a pilot study. J Clin Diagn Res, 9, ZC59.

Rai A, Kaur M, Gombra V, et al (2019). Comparative evaluation of curcumin and antioxidants in the management of oral submucous fibrosis. J Invest Clin Dentist, 10, e12464.

Rao S, Dinkar C, Vaishnav LK, et al (2014). The Indian spice turmeric delays and mitigates radiation-induced oral mucositis in patients undergoing treatment for head and neck cancer: an investigational study. Integrat Cancer Ther, 13, 201-10.

Rodríguez-Caballero A, Torres-Lagares D, Robles-García M, et al (2012). Cancer treatment-induced oral mucositis: a critical review. Int J Oral Maxillofacial Surg, 41, 225-38.

Saldanha SP, Almeida VD. (2014). A comparative study to assess the effectiveness of turmeric mouth wash versus saline mouth wash on treatment induced oral mucositis (tiom) in a selected hospital at Mangalore. J Clin Res Bioethics, 5, 1 .

Shaikh J, Ankola, DD, Beniwal V, et al (2009). Nanoparticle encapsulation improves oral bioavailability of curcumin by at least 9-fold when compared to curcumin administered with piperine as absorption enhancer. Eur J Pharm Sci, 37, 223.

Shah S, Rath H, Sharma G, Senapati SN, Mishra E (2020). Effectiveness of curcumin mouthwash on radiation-induced oral mucositis among head and neck cancer patients: A triple-blind, pilot randomised controlled trial. Indian J Dent Res, 31, 718.

Sonis ST, Elting LS, Keefe D, et al (2004). Perspectives on cancer therapy-induced mucosal injury: pathogenesis, measurement, epidemiology, and consequences for patients. Cancer: Interdisciplinary. Int J Am Cancer Soc, 100, 1995-5.

Tian X, Liu XL, Pi YP, et al (2018). Oral zinc sulfate for prevention and treatment of chemotherapy-induced oral mucositis: a meta-analysis of five randomized controlled trials. Front Oncol, 8, 484.

Van den Wyngaert T (2012). Topical honey application to reduce radiation-induced oral mucositis: a therapy too sweet to ignore?. J Evidence Based Dent Pract, 12, 203-5.

Vera-Llonch M, Oster G, Hagiwara M, et al (2006). Oral mucositis in patients undergoing radiation treatment for head and neck carcinoma: risk factors and clinical consequences. Cancer: Interdisciplinary. Int J Am Cancer Soc, 106, 329-6.

Yuan A, Sonis S (2014). Emerging therapies for the prevention and treatment of oral mucositis. Exp Opinion Emerging Drugs, 19, 343-1.

Zhang L, Tang G, Wei Z (2021). Prophylactic and therapeutic effects of curcumin on treatment-induced oral mucositis

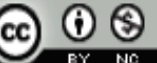

This work is licensed under a Creative Commons AttributionNon Commercial 4.0 International License. in patients with head and neck cancer: A Meta-Analysis of Randomized Controlled Trials. Nutr Cancer, 73, 740-9. 